\title{
Extensive mediastinal aspergillosis presenting with dyspnoea and cardiac tamponade symptoms
}

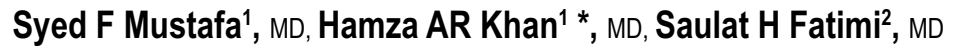 \\ ${ }^{1}$ Medical College, Aga Khan University, Karachi, Pakistan \\ ${ }^{2}$ Division of Cardiothoracic Surgery, Department of Surgery, Aga Khan University, Karachi, Pakistan \\ *Corresponding author: hamza_saeedahmed@hotmail.com
}

Hong Kong Med J 2017;23:202-3

DOI: $10.12809 / \mathrm{hkmj} 154790$

\section{Case report}

An 18-year-old girl was admitted to the Aga Khan Hospital, Pakistan in April 2014 with progressively worsening shortness of breath, orthopnoea, malaise, and low-grade fever for 3 years, and generalised body swelling for 6 months. She had no significant medical history or trauma.

On physical examination she was a mediumbuilt girl with a heart rate of 120 beats/min, raised jugular venous pressure, and bilateral pedal pitting oedema. Chest auscultation revealed muffled heart sounds with bilateral basilar lung crepitations. The chest X-ray showed wide mediastinum with clear lung markings.

The computed tomographic (CT) scan showed a conglomerate undefined mass in the anterior, middle, and posterior mediastinum encasing the entire heart and great vessels with most marked extension in the retrocardiac area (Fig 1). The echo showed a large echo-dense mass compressing the heart with invasion into the left atrium (LA), obliterating most of the LA cavity.

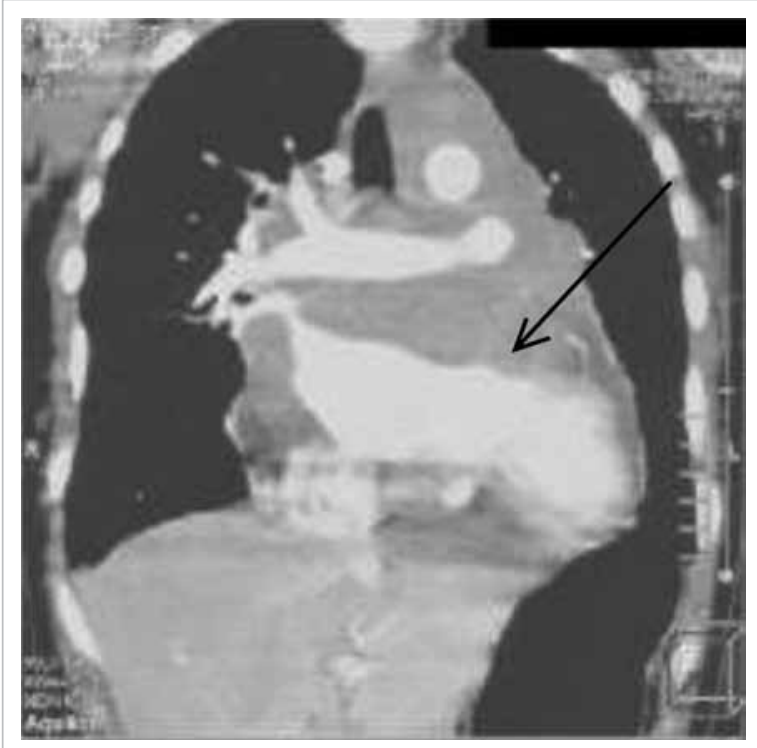

FIG I. Computed tomographic scan showing an undefined mass (arrow) in the anterior, middle, and posterior mediastinum encasing the entire heart and great vessels
Video-assisted thoracoscopic surgery was performed and the mass biopsied for histopathological examination. The histopathology showed fibrosis and chronic granulomatous inflammation with fungal hyphae on periodic acidSchiff staining (Fig 2). The fungal cultures grew Aspergillus flavus.

A diagnosis of invasive mediastinal aspergillosis was made and the patient was started on oral itraconazole $100 \mathrm{mg}$ twice a day. She showed a remarkable recovery in her symptomatology in the first 6 weeks of follow-up.

\section{Discussion}

Aspergillosis refers to a variety of diseases caused by several species of Aspergillus organisms that are abundant in the environment and recognition of which has increased over recent years. There are three main categories in general: saprophytic aspergilloma, allergic bronchopulmonary aspergillosis, and invasive aspergillosis. The term 'invasive aspergillosis' is generally used to imply a

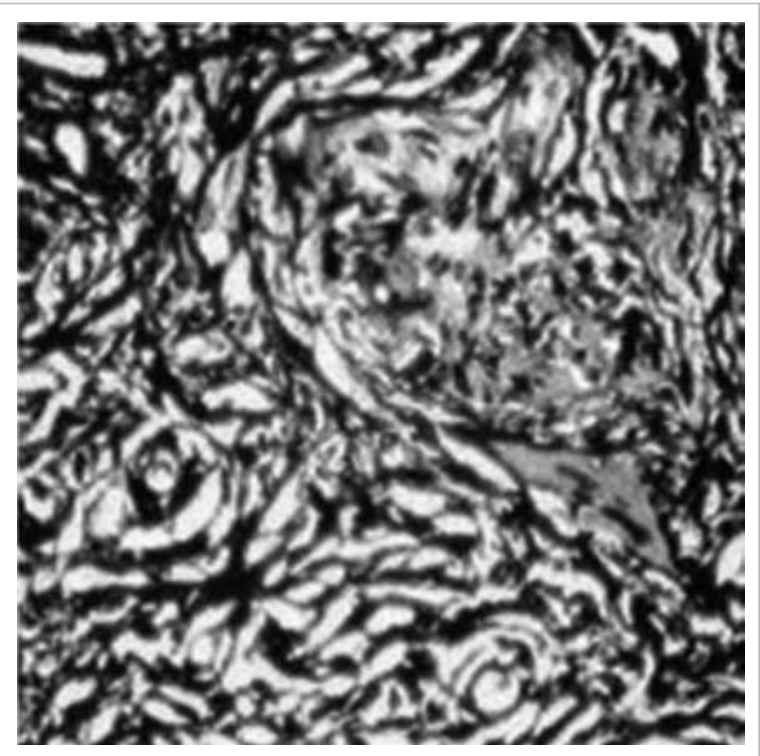

FIG 2. Histopathology showing fibrosis and chronic granulomatous inflammation along with fungal hyphae stained with periodic acid-Schiff stain (original magnification, $x$ 400) 
histopathologically demonstrated invasion of tissues by these spores. It represents an important cause of morbidity and mortality, particularly among immunocompromised patients, occurring mostly in those with haematological malignancies who are undergoing chemotherapy and organ transplantation with concomitant immunosuppressive therapy. Extension of invasive pulmonary aspergillosis to the mediastinum has been reported only rarely. ${ }^{1}$ Our case is an immunocompetent patient with extensive mediastinal aspergillosis who presented with dyspnoea and symptoms of cardiac tamponade.

Invasive aspergillosis varies in severity and clinical course, depending upon the affected organ and the host. It is caused by many Aspergillus groups; however the most common is the Aspergillus fumigatus group. These groups live in soil, and derive nutrients from dead plants and animal matter. It has been further subdivided in recent years to angioinvasive and airway-invasive aspergillosis. Invasive aspergillosis is a major cause of morbidity and mortality in immunosuppressed patients. ${ }^{2}$

The form that Aspergillus lung disease takes is heavily dependent on the immune response of the patient. ${ }^{3}$ Indolent forms of locally invasive aspergillosis in the form of chronic necrotising or semi-invasive aspergillosis in apparently immunocompetent individuals are now recognised.

Invasive aspergillosis is a result of three factors: (1) suppression of the immune response due to debilitating disease or therapy; (2) glucocorticoid therapy with consequent diminished inflammatory response and disruption of the normal flora by antimicrobial agents ${ }^{4}$; and (3) local implantation of the fungus. The disease is well known in immunocompromised individuals but has also been described in healthy individuals. Patients with a compromised immune system, specifically with neutropenia, are susceptible to acute airway-invasive and angioinvasive aspergillosis in the absence of preexisting lung pathology.

There have been a few cases described in which patients were immunocompetent. Orr et $\mathrm{al}^{5}$ documented two patients with normal host defences in whom postmortem examination revealed death due to disseminated aspergillosis (one patient had a history of coronary heart disease, the other acute renal failure following gangrenous appendicitis). Our patient was otherwise healthy as confirmed by the normal haematological studies.

The usual pathogenesis of invasive aspergillosis is by dissemination from a primary site, such as the lungs or the paranasal sinuses, or by contiguous spread. ${ }^{6}$ In this particular patient, the chest X-ray and CT scan of the chest were clear without a primary lesion. It was therefore postulated that inhalation of the fungal spores with immediate mediastinal invasion resulted in the implantation of a high concentration of aspergillus spores around the heart. This was followed by chronic infiltration around the pericardium and extension into the heart.

This idea was supported by the histopathology report of granulomatous tissue fibrosis, indicating a long-standing process, and in keeping with the history of worsening shortness of breath for 3 years. Our case showed an undefined mass in the anterior, middle, and posterior mediastinum extending into the LA. The effects of the mass could explain all of the patient's symptoms, namely worsening dyspnoea and cardiac tamponade symptoms.

In conclusion, this case highlights that there is usually a long dormant period before the clinical manifestation of aspergillosis, clinically and radiologically the disease may be suggestive of a malignant process, and contamination with aspergillus should be considered from all possible aspects, particularly in healthy individuals.

\section{References}

1. Shakoor MT, Ayub S, Ayub Z, Mahmood F. Fulminant invasive aspergillosis of the mediastinum in an immunocompetent host: a case report. J Med Case Rep 2012;6:311.

2. Al-Alawi A, Ryan CF, Flint JD, Müller NL. Aspergillusrelated lung disease. Can Respir J 2005;12:377-87.

3. Gefter WB. The spectrum of pulmonary aspergillosis. J Thorac Imaging 1992;7:56-74.

4. Mashimoto H, Suyama N, Araki J, et al. A case of mediastinitis and bilateral pyothorax, following acute epiglottitis with concurrent Aspergillus infection [in Japanese]. Kansenshogaku Zasshi 1992;66:648-52.

5. Orr DP, Myerowitz RL, Dubois PJ. Patho-radiologic correlation of invasive pulmonary aspergillosis in the compromised host. Cancer 1978;41:2028-39.

6. Hendrix WC, Arruda LK, Platts-Mills TA, Haworth CS, Jabour R, Ward GW Jr. Aspergillus epidural abscess and cord compression in a patient with aspergilloma and empyema. Survival and response to high dose systemic amphotericin therapy. Am Rev Respir Dis 1992;145:14836. 